\title{
Electroencephalographic changes as neuroscience-based cognitive skill training program
}

\author{
Phakkharawat Sittiprapaporn \\ Assistant Professor and Head, Brain Science and Engineering Innovation Research Group, School of Anti-Aging and \\ Regenerative Medicine, Mae Fah Luang University, Bangkok, Thailand, and Department of Anti-Aging Science, School \\ of Anti-Aging and Regenerative Medicine, Mae Fah Luang University, Bangkok, Thailand
}

Background: Brain is an organ that is easily affected by the physical changes. Abnormalities are known to occur with the brain in terms of memory, eyesight, intellect, and balance as the brain is an organ that is easily affected by the physical changes. Executive functions have been shown to be related to academic achievement and the ability to learn, as well as a range of a neuro developmental disorders. The failure of many traditional educational interventions for students with specific learning disabilities may be attributed to large deficits in executive functions, including working memory and inhibition. Aims and Objective: This study examined how BrainWare SAFARI, a computer-based training, aided students with specific learning disabilities in enhancing their executive functions and specific learning disabilities indexed by attention and meditation levels. Materials and Methods: The present experimental study examined the effectiveness of a specific 12-week neuroscience-based, cognitive skills computertraining program, BrainWare SAFARI, on the cognitive processing of elementary students with specific learning disabilities. Results: The results of this study showed that all participants were able to complete only some sessions in BrainWare SAFARI experienced significant improvements in multiple areas of cognitive processing. The results also showed that the attention level changed with statistically significant while the meditation level was changed with no statistically significant. Conclusion: Thus, this neuroscience-based, cognitive skills computer-training program, BrainWare SAFARI, could result in increased academic performance.

Key words: Brain; Cognitive Skills; Cognitive Deficits; Learning Disabilities; Children

\section{INTRODUCTION}

Brain is an organ that is easily affected by the physical changes, especially in the modern time when people are easily poisoned by the increasing pollution. When the poisonous substances enter the body, every organ, including the brain will be endangered. Abnormalities will occur to the brain in terms of memory, eyesight, intellect, and balance. Because of these damages, people should know how to protect themselves from the poisonous substances. ${ }^{1-3}$ Abnormalities are also known to occur with the brain in terms of memory, eyesight, intellect, and balance as the brain is an organ that is easily affected by the physical changes. ${ }^{1-2}$
Executive functions have been shown to be related to academic achievement and the ability to learn, as well as a range of a neuro developmental disorders. The failure of many traditional educational interventions for students with specific learning disabilities may be attributed to large deficits in executive functions, including working memory and inhibition. Understanding the role of executive function may offer new learning opportunities for children facing educational barriers due to specific learning disabilities. Working memory, executive functions, and cognitive processes associated with specific academic areas, are empirically identified as being the core underlying cognitive deficits in students with specific learning disabilities. 
BrainWare SAFARI is a software program which is designed to comprehensively develop the cognitive skills in children. These cognitive skills are most important for learning. BrainWare SAFARI delivers cognitive skills in a fun and entertaining video-game format. Several previous published researches showed that students who used BrainWare SAFARI experienced significant improvements in their cognitive skills. In addition, a growing body of research shows the connection between the development of cognitive skills and academic progress. Academic progress typically lags cognitive growth and the rate of gain in academic achievement is highly individual. Moreover, some students experienced an immediate lift in academic scores, while others might have significant catching-up to do because they need to learn or re-learn material they missed.

Electroencephalography (EEG) is traditionally used to measure the brainwaves. Each type of brainwave is associated with one's state of consciousness and different mood state. ${ }^{47}$ From all types of brainwave, beta brainwave is seen in normal activities as well as stressful conditions or difficulties in mental concentration. On the other hand, alpha brainwave is seen in wakefulness, relaxed, effortless and alertness condition. Therefore, persons with high alpha brainwave and low in beta brainwave could indicate status of relaxation, arousal, less stress and better concentration. ${ }^{8-12}$ Therefore, this study was set to find data by using the effectiveness of commercially available lightweight electroencephalographic (EEG) device, NeuroSky's Mindwave Mobile. So, the purpose of this study was to determine the efficacy of BrainWare SAFARI game brain training for cognitive performance and electroencephalographic activities in Thai students with specific learning disabilities. The measurement of electroencephalographic activities of BrainWare SAFARI game brain training process was mostly done by letting the students practiced and then measured the electroencephalographic activities.

\section{MATERIALS AND METHODS}

\section{Participants}

The experiment was done with 20 students, aged between 5-8 years old, who were students with specific learning disabilities, but no record of brain surgery, not taking medicines or drugs that affect to nervous system. Participants were excluded if they had the following criteria: left handedness; history of medical and neurologic diseases; psychiatric disorders; head trauma; assumption of Central Nervous System active drugs in the two weeks prior to study entry; and presence of EEG abnormalities at the baseline recording, respectively.
All participants' parents provided written consent to participate in the study after receiving information about the aims of the study.

\section{Tools and equipment}

Recording personal information, namely, age, gender, nationality, and history of illness were recorded. The electroencephalographic recording was applied in this study. The neuro headset was used to display the output, analyze and record electroencephalographic activities. The principles of analytical check were based on the 10-20 system or International 10-20 system by displaying the electroencephalographic output. Electroencephalographic frequency analysis was performed by means of a Fast Fourier Transform (FFT) algorithm, with a 2-second interval on the electroencephalographic signal. The following frequency bands were considered: delta wave $(0.1-3.0 \mathrm{~Hz})$; theta wave $(4.0-7.0 \mathrm{~Hz})$; alpha wave $(8.0-12.0 \mathrm{~Hz})$; beta wave $(13.0-30.0 \mathrm{~Hz})$; and gamma wave $(30.0-60.0 \mathrm{~Hz})$, respectively.

In this study, the effectiveness of commercially available lightweight electroencephalographic devices, NeuroSky's Mindwave Mobile, was applied. Via the application of a single electrode and signal-processing unit in a headband arrangement, the NeuroSky's Mindwave Mobile provided two 100-state outputs operating at $1 \mathrm{~Hz}$. Although the NeuroSky's Mindwave Mobile provided a much coarser picture of brain activity than multi-electrode electroencephalographic device or other technologies, the principle advantage of the NeuroSky's Mindwave Mobile was its unobtrusive nature, which minimizes the aforementioned difficulties in conducting accurate user studies.

\section{Spatial ability test}

Spatial ability test used in this study is known as the test to measure capacity to understand, reason and remember the spatial relations among objects or space. ${ }^{13}$ Spatial or visuo-spatial perception, spatial visualization, mental folding and mental rotation are common types of spatial abilities. However, only spatial visualization is known to be characterized as complicated multi-step manipulations of spatially presented information. In the same way, spatial working memory is known as the ability to temporarily store a certain amount of visual-spatial memories under attentional control in order to complete a task. ${ }^{14-15}$ Each of four main spatial abilities theoretically have unique properties and importance to many types of tasks whether in certain jobs or everyday life. ${ }^{13}$

\section{Cognitive training program}

BrainWare SAFARI was a cognitive learning program which was designed to comprehensively develop the cognitive skills. These cognitive skills are important for 
learning and the BrainWare SAFARI program delivered in a fun and entertaining video-game format. BrainWare SAFARI consisted of 20 exercises designed to develop various cognitive skills. In 18 of the exercises, each with seven levels, levels were progressively more difficult. Each level must be passed before the student could progress to the next level. The two logic and reasoning exercises consisted of 21 levels each and were of approximately the same level of difficulty, allowing the students to see the right answer and move on after two incorrect answers (Figure 1). BrainWare SAFARI required an internet connection. It was designed as a rich-client application to enhance the user's experience, but log-in and progress through the program are controlled by LEC's server. This system enabled the player to always begin in the exercises where he or she left off, regardless of whether he or she is on the same computer. BrainWare SAFARI program is developed by BrainWare Learning Company and more details of BrainWare SAFARI can be seen at www.mybrainware.com.

\section{Electroencephalographic recording}

Measuring electroencephalographic activities while performing spatial ability tests run by SuperLab Pro. Version 2.0 (Cedrus Corporation, San Pedro, CA, USA), stimuli presentation, compared to baseline. SuperLab Pro was used to develop spatial ability tests for electroencephalographic measurement. A single-channel electroencephalogram was designed to collect brain activity data from the students' brain. Simplicity and efficiency were the main reasons for designing a single-channel electroencephalographic monitoring system. Electroencephalographic data was analyzed offline and the following frequency bands were computed: delta wave ranging from 0.1 to $3.0 \mathrm{~Hz}$, theta wave $(4.0$ to $7.0 \mathrm{~Hz})$, alpha wave $(8.0$ to $13.0 \mathrm{~Hz})$, beta wave $(14.0$ to $30.0 \mathrm{~Hz})$, and gamma wave $(31.0$ to $47.0 \mathrm{~Hz})$, respectively.

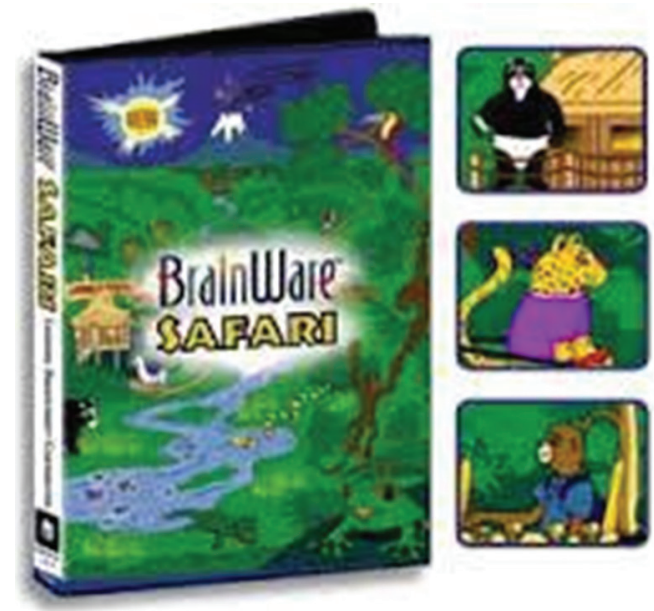

Figure 1: BrainWare SAFARI Program by BrainWare Learning Company

\section{Statistical analysis}

The quantitative data was analyzed by mean of average and standard deviation. Using inferential statistical analysis to compare each type of brainwaves by compared t-test. Every test was set to the statistic significant at $p<0.05$.

\section{RESULTS}

In this study, the attention and mediation levels were measured both before and after training all exercises in the Brain Ware SAFARI cognitive skills training program. The results showed that the attention level changed in a statistically significant at 0.05 level $(p$-value $=0.04)$. The average before training was $60.51( \pm 1.49)$ score while the average after training was 70.35 ( \pm 1.33$)$ score. For meditation level, there was changed with no statistically significant at 0.05 level $(p$-value $=0.72)$. The average before training was $40.92( \pm 1.73)$ score and the average after training was 50.47 ( \pm 1.26) score (Figure 2$)$.

Based on Table 1 and Figure 3, it was found that theta and alpha brainwaves gradually increased while delta and beta brainwaves did not change. Theta and alpha brainwaves increased with statistically significant at the 0.05 level while performing spatial ability tasks. On the other hand, only gamma brainwave was found to be decreased while performing spatial ability tasks. In addition, it was found that delta brainwave did not change much while performing spatial ability tasks after BrainWare SAFARI training compared to baseline (before training: $0.02 \pm 0.057 \mu \mathrm{V}$; after training: $0.036 \pm 0.015 \mu \mathrm{V}$; $\mathrm{t}(19)=1.6 ; p=0.29)$ while theta brainwave increased with statistical significant while performing spatial ability tasks after BrainWare SAFARI training compared to baseline (before training: $0.27 \pm 0.17 \mu \mathrm{V}$; after training: $1.18 \pm 0.09 \mu \mathrm{V}$; $\mathrm{t}(19)=1.3 ; p=<0.05)$.

In the same way, it was found that alpha brainwave was found to be increased with statistically significant at the 0.05 level while performing spatial ability tasks after BrainWare SAFARI training compared to baseline (before training: $0.14 \pm 0.38 \mu \mathrm{V}$; after training: $1.06 \pm 0.02 \mu \mathrm{V} ; \mathrm{t}(19)=0.47 ; p=0.01)$. Similar to alpha brainwave, beta brainwave increased with no statistically significant while performing spatial ability tasks after BrainWare SAFARI training compared to baseline (before training: $0.18 \pm 0.01 \mu \mathrm{V}$; after training: $0.22 \pm 0.31 \mu \mathrm{V} ; \mathrm{t}(19)=1.17 ; \mathrm{p}=0.34)$. Finally, it was found that gamma brainwave decreased with no statistically significant while performing spatial ability tasks after BrainWare SAFARI training compared to baseline (before training: $0.021 \pm 0.08 \mu \mathrm{V}$; after training: $0.14 \pm 0.25 \mu \mathrm{V} ; \mathrm{t}(19)=1.14 ; p=0.37)$. 


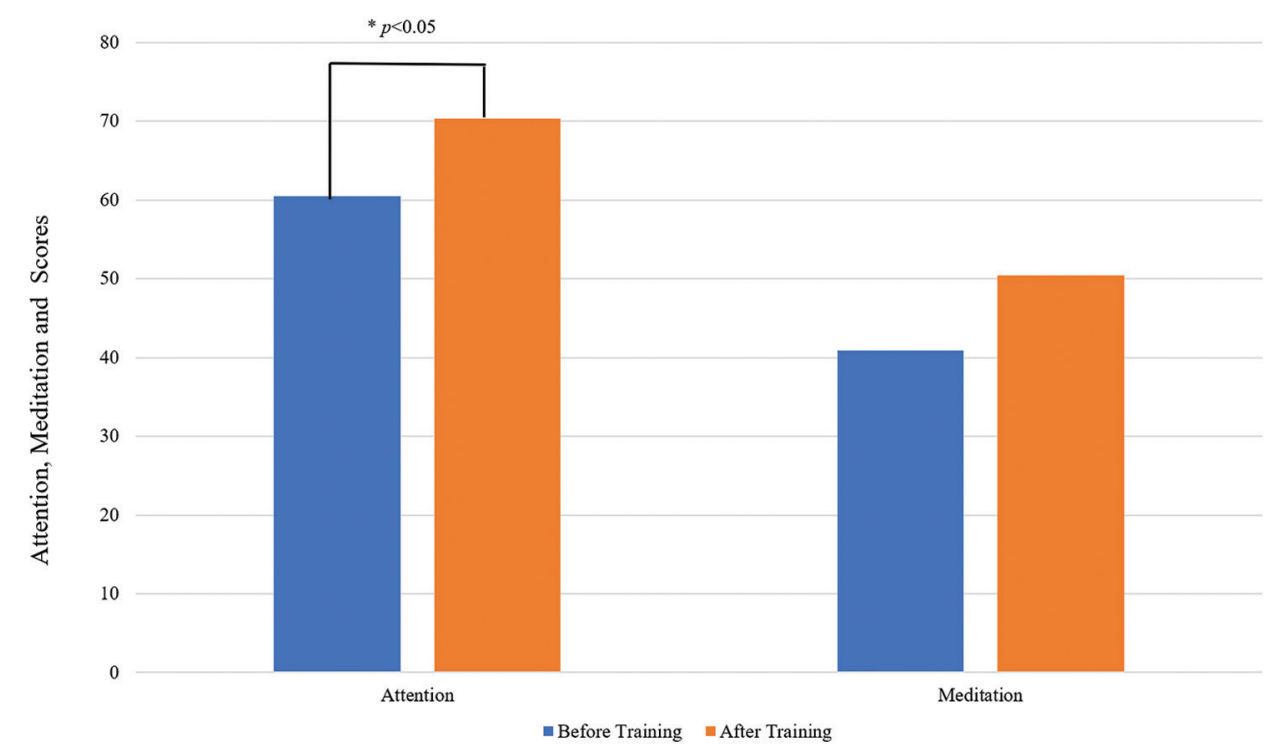

Figure 2: Attention and mediation levels were measured both before and after training with all exercises in the BrainWare SAFARI program

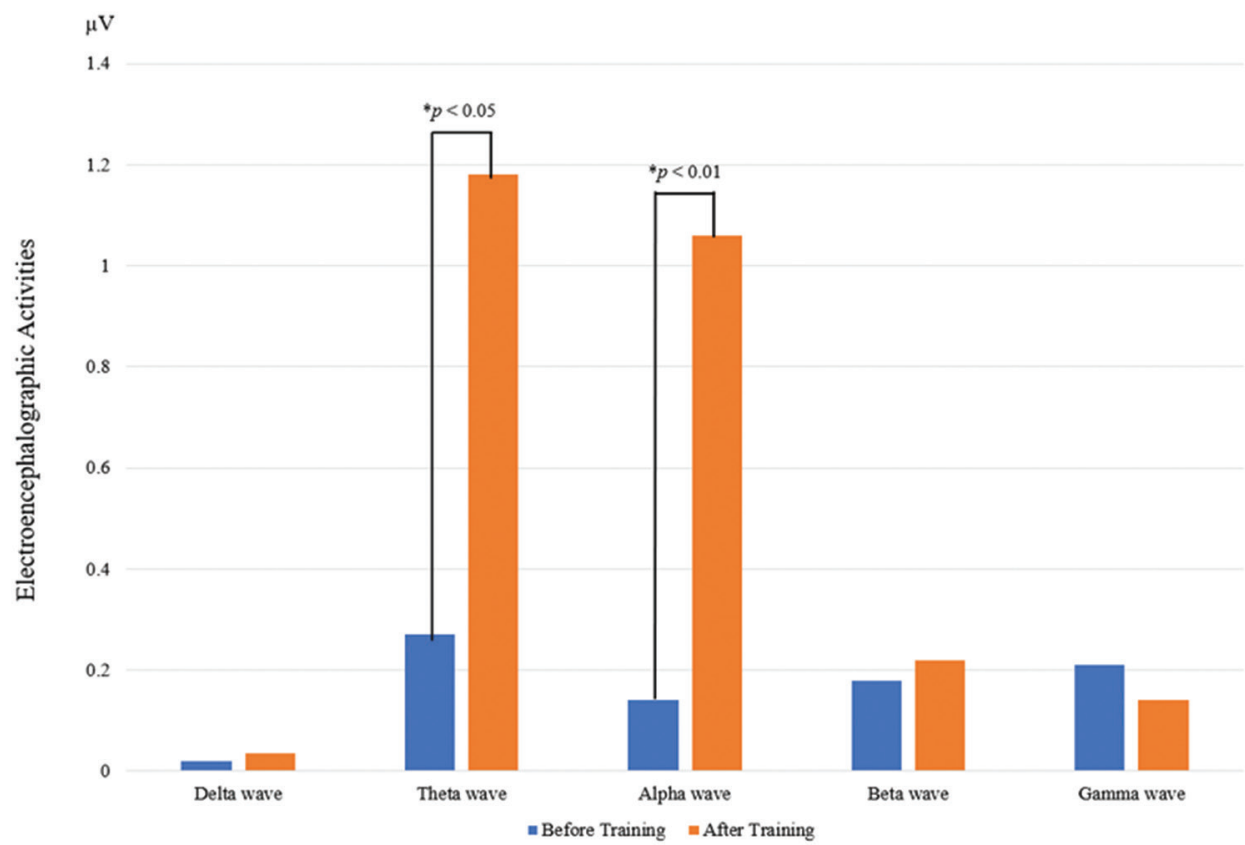

Figure 3: Electroencephalographic activities while performing the spatial ability tasks both before and after BrainWare SAFARI training program

\begin{tabular}{|c|c|c|c|c|c|}
\hline \multirow[t]{2}{*}{ Electroencephalographic activities } & \multicolumn{2}{|c|}{ Before training } & \multicolumn{2}{|c|}{ After training } & \multirow[t]{2}{*}{$p$-value } \\
\hline & Mean & SD & Mean & SD & \\
\hline Delta brainwave & 0.02 & 0.57 & 0.036 & 0.15 & 0.29 \\
\hline Theta brainwave & 0.27 & 0.17 & 1.18 & 0.09 & $0.05^{\star}$ \\
\hline Alpha brainwave & 0.14 & 0.38 & 1.06 & 0.02 & $0.01^{*}$ \\
\hline Beta brainwave & 0.18 & 0.01 & 0.22 & 0.31 & 0.34 \\
\hline Gamma brainwave & 0.021 & 0.08 & 0.14 & 0.25 & 0.37 \\
\hline
\end{tabular}




\section{DISCUSSION}

Participants were able to complete some sessions in BrainWare SAFARI training program showing some improvements in multiple areas of cognitive processing. Additionally, while the attention level changed with statistically significant, the meditation level was changed with no statistically significant.

Similar to Lumosity, a web-based brain fitness training that previous study reported the improve visual attention and memory recall in healthy adults after practicing. ${ }^{16}$ Some previous studies reported that Lumosity could improve visual attention in mild cognitive impairment patients, and improved speed, flexibility and memory recall in cancer recovered child patient. ${ }^{17-18}$ BrainWare SAFARI is a game and brain training program used in many researches that show significant result in improving cognitive performance including speed, memory, spatial ability, and problem solving, respectively. Theoretically, spatial abilities were called as the mental capacities involving with construction, transformation and interpretation of mental images. ${ }^{19}$ These abilities reflected the use of mental images for manipulating spatial representations. ${ }^{19}$ Several studies have been tried to determine the different factors composing the spatial abilities. The visualization, orientation and spatial relations were also known as main factors. ${ }^{19}$ Spatial ability test is known as the test to measure capacity to understand, reason and remember the spatial relations among objects or space. ${ }^{13}$ Spatial or visuo-spatial perception, spatial visualization, mental folding and mental rotation are common types of spatial abilities. Each of four main spatial abilities theoretically have unique properties and importance to many types of tasks whether in certain jobs or everyday life. ${ }^{13}$ The spatial relation ability corresponds to the capacity to rapidly and accurately rotate a mental image. ${ }^{19}$ The spatial abilities are linked with the mental imagery including the mental rotation task and the spatial relation which are intimately related as rapidly rotate a mental image while performing this task. ${ }^{19-20}$ These links between spatial abilities and the mental imagery could affect to the mental images (MI)-Brain Computer Interface (BCI) performance. ${ }^{20}$

A brain computer interface (BCI) is a hardware and software communication system that enables its user to interact with surroundings without the involvement of peripheral nerves and muscles, i.e., by using control signals generated from electroencephalographic activity. ${ }^{1}$ Participants in the present study showed some mean improvements in electroencephalographic activities and some areas of cognitive functions including memory, attention, flexibility and problem solving, respectively. This was consistent with findings in several previous studies such as in healthy adults, older adults with mild cognitive impairment, and chemotherapy-treated cancer survivors. ${ }^{16-18}$ The electroencephalographic activities during practicing attention via BrainWare SAFARI game brain training showed significant increase in theta and alpha frequency bands. This study was consistent with the following previous studies i.e., Egner and Gruzelier ${ }^{21}$ and Rossiter and LaVaque ${ }^{22}$ found that SMR (Sensorimotor Rhythm) or lower beta related to attention.

Cognitive skill training especially spatial ability, has been shown to be efficient in many different areas including mathematics and engineering education. ${ }^{23}$ In addition, both theta and alpha brainwaves were gradually increased after practicing all exercises in the BrainWare SAFARI program. The results stayed in the line with previous studies showing that brain training program has efficient in many different areas including mathematics and engineering education. ${ }^{23}$ Moreover, brain and spatial ability trainings were shown to impact capacities in scientific learning. ${ }^{24} \mathrm{~A}$ well-known of mathematics and spatial abilities training was based on the Vanderberg and Kuse ${ }^{23}$ who developed the Mental Rotation test. The mental rotation test was then developed by Hoyek et al..$^{25}$ using a computerized version to train students' spatial abilities.

\section{CONCLUSION}

The findings in this study revealed that both theta and alpha brainwaves increased with statistically significant after training by BrainWare SAFARI training program. The mean improvement of cognitive skills including speed, memory, attention, flexibility and problem solving shown by participants were consistent with those findings in several previous studies. By practicing attention games as BrainWare SAFARI game brain training could significantly increase theta and alpha frequency bands. BrainWare SAFARI game brain training could significantly improve cognitive performance indexed by the electroencephalographic activities.

\section{ACKNOWLEDGEMENT}

This research was supported by research funding from Asia Research Center, Chulalongkorn University, Bangkok, Thailand, and The Korea Foundation for Advanced Studies (KFAS), Korea. Authors gratefully acknowledge Mae Fah Luang University grant (Electroencephalogram Laboratory 2019), Mae Fah Luang University grant (MFU-grant no. 2201A601814), and Brain Science and Engineering Innovation Research Group, Mae Fah Luang University grant (2019) (MFU-grant no. 611U109005) and 2020, Thailand, for the support and necessary provision. 
We thank all of the subjects involved in this research as main data provider of this studies.

\section{REFERENCES}

1. Bailey SP, Hall EE, Folger A and Miller PC. Changes in EEG during graded exercise on a recumbent cycle ergometer. J Sports Sci Med 2008; 7: 505-511.

2. Gutmann B, Mierau A, Hulsdunker T, Hildebrand C, Przyklenk A, Hollmann W, et al. Effects of Physical Exercise on Individual Resting State EEG Alpha Peak Frequency. Neural Plast 2015; 717312.

3. Moraes $H$, Deslandes A, Silveira $H$, Ribeiro $P$, Cagy $M$, Piedade R,et al. The effect of acute effort on EEG in healthy young and elderly subjects. Eur J Appl Physiol 2011; 111(1): 67-75.

4. Allen JJB, Coan JA and Nazarian M. Issues and assumptions on the road from raw signals to metrics of frontal EEG asymmetry in emotion. Biol. Psychol 2004; 67: 183-218.

5. Crabbe JB and Dishman RK. Brain electrocortical activity during and after exercise: A quantitive synthesis. Psychophysiol 2004; 41: 563-574.

6. Davis JM and Bailey SP. Possible mechanisms of central nervous system fatigue during exercise. Med. Sci. Sport Exer 1997; 29(1): 45-57.

7. Kakizaki T. Effects of bicycle exercise on occipital EEG amplitude in male students. Industrial Health 1988; 26: 191-195.

8. Kamijo K, Nishihira Y, Hatta A, Kaneda T, Kida T, Higashiura T, et al. Changes in arousal level by differential exercise intensity. Clin Neurophysiol 2004; 115: 2693-2698.

9. Kraaier V, Van Huffelen AC and Wieneke GH. Changes in quantitative EEG and blood flow velocity due to standardized hyperventilation; a model of transient ischaemia in young human subjects. Electroencephal. Clin. Neurophysiol 1988; 70: 377-387.

10. Kraaier V, Van Huffelen AC, Wieneke GH, Van der Worp HB and Bär PB. Quantitative EEG changes due to cerebral vasoconstriction. Indomethacin versus hyperventilationinduced reduction in cerebral blood flow in normal subjects. Electroencephal Clin Neurophysiol 1992; 82: 208-212.

11. Kubitz KA and Mott AA. EEG power spectral densities during and after cycle ergometer exercise. Res Quart Exer Sport 1996; 67(1): 91-96.

12. Robertson IH, Manly T, Andrade J, Baddeley BT and Yiend J.
Oops!: Performance correlates of everyday attentional failures in traumatic brain injured and normal subjects. Neuropsychol 1997; 35(6): 747-758.

13. Donnon T, Jean-Gaston $\mathrm{D}$ and Claudio V. Impact of cognitive imaging and sex differences on the development of laparoscopic suturing skills. Canadian J Surg 2005; 48(5): 387-393.

14. Linn $M C$ and Petersen $A C$. Emergence and Characterization of Sex Differences in Spatial Ability: A Meta-Analysis. Child Dev1990; 56(6): 1479-1498.

15. Shelton JT, Elliott EM, Hill BD, Calamia MR and Gouvier WMD. A Comparison of Laboratory and Clinical Working Memory Tests and Their Prediction of Fluid Intelligence. Intelligence 2009; 37(3): 283.

16. Joshep HL, David D, Kumal S, Gregory K and Michael S. Enhancing visual attention and working memory with a webbased cognitive training program. Mensa Res 2010; 42(2): 13-20.

17. Finn $\mathrm{M}$ and $\mathrm{McDonald} \mathrm{J}$. Improvement in visual sustained attention in older adults with mild cognitive impairment, Australian J Ageing 2010; 29(Supplement 2): 15.

18. Kessler $S$, Hosseini $H$, Heckler $C$, Janelsins $M$, Palesh $O$, Mustian $\mathrm{K}$, et al. Cognitive Training for Improving Executive Function in Chemotherapy-Treated Breast Cancer Survivors. Clin Breast Cancer 2013; 13(4): 299-306.

19. Poltrock $S E$ and Brown $P$. Individual differences in visual imagery and spatial ability. Intelligence 1984; 8: 93-138.

20. Rourke BP and Finlayson MAJ. Neuropsychological significance of variations in patterns of academic performance: Verbal and visual spatial abilities. J Abn Child Psycho 1978; 121-133.

21. Egner $\mathrm{T}$ and Gruzelier $\mathrm{JH}$. Learned self-regulation of EEG frequency components affects attention and event-related brain potentials in humans. Neuro Report 2001; 12: 4155-4159.

22. Rossiter R and LaVaque TJ. A comparison of EEG biofeedback and psychostimulants in treating attention deficit hyperactivity disorders. J Neurother 1995; 4: 48-59.

23. Vandenberg SG and Kuse AR. Mental rotations, a group test of three-dimensional spatial visualization. Percept Motor Skills 1978; 47: 599-604.

24. Bishop AJ. Spatial abilities and mathematics education - a review," Edu. Studies Math 1980; 11: 257-269.

25. Hoyek N, Collet C, Rastello O, Fargier P, Thiriet P and Guillot A. Enhancement of mental rotation abilities and its effect on anatomy learning. Teaching and Learning Med 2009; 21: 201-206.

\footnotetext{
Authors Contribution:

PS- Concept and design of the study, statistically analyzed and interpreted, manuscript preparation, critical revision of the manuscript.

Work attributed to:

Brain Science and Engineering Innovation Research Group, School of Anti-Aging and Regenerative Medicine, Mae Fah Luang University, Thailand.

Orcid ID:

Dr. Phakkharawat Sittiprapaporn - (1) https://orcid.org/0000-0002-4103-9396

Source of Support: Asia Research Center, Chulalongkorn University, Bangkok, Thailand, and The Korea Foundation for Advanced Studies (KFAS), Korea; Mae Fah Luang University grant (Electroencephalogram Laboratory 2019), Mae Fah Luang University grant (MFU-grant no. 2201A601814), and Brain Science and Engineering Innovation Research Group, Mae Fah Luang University grant (2019) (MFU-grant no. 611U109005) and 2020, Thailand, Conflict of Interest: None declared.
} 